\title{
Dielectric dependence of field-induced interspherical force
}

\author{
Zhiyong Wang ${ }^{1}$, Rong Shen ${ }^{1}$, Xiaojuan Niu ${ }^{1}$, Gang Sun ${ }^{1}$, \\ Kunquan Lu ${ }^{1}$, Bo Hou ${ }^{2}$ and Weijia Wen ${ }^{2,3}$ \\ ${ }^{1}$ Laboratory of Soft Matter Physics, Institute of Physics, Chinese Academy of Sciences, \\ Beijing, People's Republic of China \\ ${ }^{2}$ Department of Physics, The Hong Kong University of Science and Technology, \\ Clear Water Bay, Kowloon, Hong Kong, People's Republic of China \\ E-mail: phwen@ust.hk
}

Received 2 November 2004, in final form 18 January 2005

Published 1 April 2005

Online at stacks.iop.org/JPhysD/38/1325

\begin{abstract}
The influence of the dielectric properties of spheres on the field-induced interspherical force is reported. We found that a higher dielectric constant gives rise to stronger interaction forces between dielectric spheres in a pair of barium titanate spheres at the Curie temperature where the abnormal dielectric property exists. The experiment indicates that, at the Curie temperature, there is an obvious peak in the interaction force due solely to the changes in the dielectric constant. In addition to the sphere's dielectric property, the dielectric property of the surrounding medium is a critical parameter associated with the interaction force between dielectric spheres. In addition, the interaction force is dependent on the frequency of the electric field.
\end{abstract}

\section{Introduction}

An electrorheological (ER) fluid, a kind of suspension mainly consisting of dielectric particles $\left(\varepsilon_{\mathrm{p}}, \sigma_{\mathrm{p}}\right)$ suspended in a nonconducting liquid $\left(\varepsilon_{\mathrm{m}}, \sigma_{\mathrm{m}}\right)$, can change from liquid to solid abruptly under an applied electric field due to interactions among the particles [1-3]. So far, there have been a number of theoretical models and experiments reported that investigate these particle-particle forces [4-9]. The dipole approximation model has often been employed to explain the ER effect in the past decade. In this model, the interparticle force, $f$, satisfies the relationship $f \sim \varepsilon_{\mathrm{m}} \beta^{2} E_{0}^{2}$, where $E_{0}$ is the imposed electric field and $\beta^{2}$ is the dielectric mismatch presented as $\left[\left(\sigma_{\mathrm{p}}-\sigma_{\mathrm{m}}\right)^{2}+\omega^{2} \varepsilon_{0}^{2}\left(\varepsilon_{\mathrm{p}}-\varepsilon_{\mathrm{m}}\right)^{2}\right] /\left[\left(\sigma_{\mathrm{p}}+2 \sigma_{\mathrm{m}}\right)^{2}+\omega^{2} \varepsilon_{0}^{2}\left(\varepsilon_{\mathrm{p}}+2 \varepsilon_{\mathrm{m}}\right)^{2}\right]$, in which the interaction force between two spheres is strongly associated with the dielectric properties of both the solid and medium phases $[4,8]$. In this paper, we investigate the influence of the dielectric properties of both the solid and medium phases on the interaction among the dielectric spheres. A pair of identical $\mathrm{BaTiO}_{3}$ spheres are first employed to study these influences under different temperatures. Since $\mathrm{BaTiO}_{3}$ has a low Curie temperature, $T_{\mathrm{c}}$, at which the permittivity rises to its maximum value according to the Curie-Weiss law,

3 Author to whom any correspondence should be addressed. the change in the permittivity of $\mathrm{BaTiO}_{3}$ with temperature yields reliable experimental observations for studying the dielectric dependence of the particle-particle forces as all other environmental factors are established for these spheres except for their permittivity.

\section{Experimental method}

The dielectric properties of barium titanate were determined with a round-flaked piece of $\mathrm{BaTiO}_{3}(2.0 \mathrm{~mm}$ thickness and $12.84 \mathrm{~mm}$ diameter) on which silver paint was coated on both surfaces to form electrodes. An HP 4192A LF impedance analyser was used to measure the capacitance and the conductance of the dielectric material at frequencies ranging from $100 \mathrm{~Hz}$ to $1 \mathrm{KHz}$. The interaction forces between the spheres were measured using a pair of identical dielectric spheres $(6.3 \mathrm{~mm}$ in diameter for all spheres used), which were sandwiched between two parallel copper electrodes in which the gaps of the two electrodes were adjusted using a computer-controlled elevator (with an accuracy of $0.001 \mathrm{~mm}$ ) and the field-induced interaction force of the two spheres was determined using an electronic balance (sensitivity of $0.001 \mathrm{~g}$ ). A temperature-controlled oil bath was used to control the temperature of the environment of the samples with an 

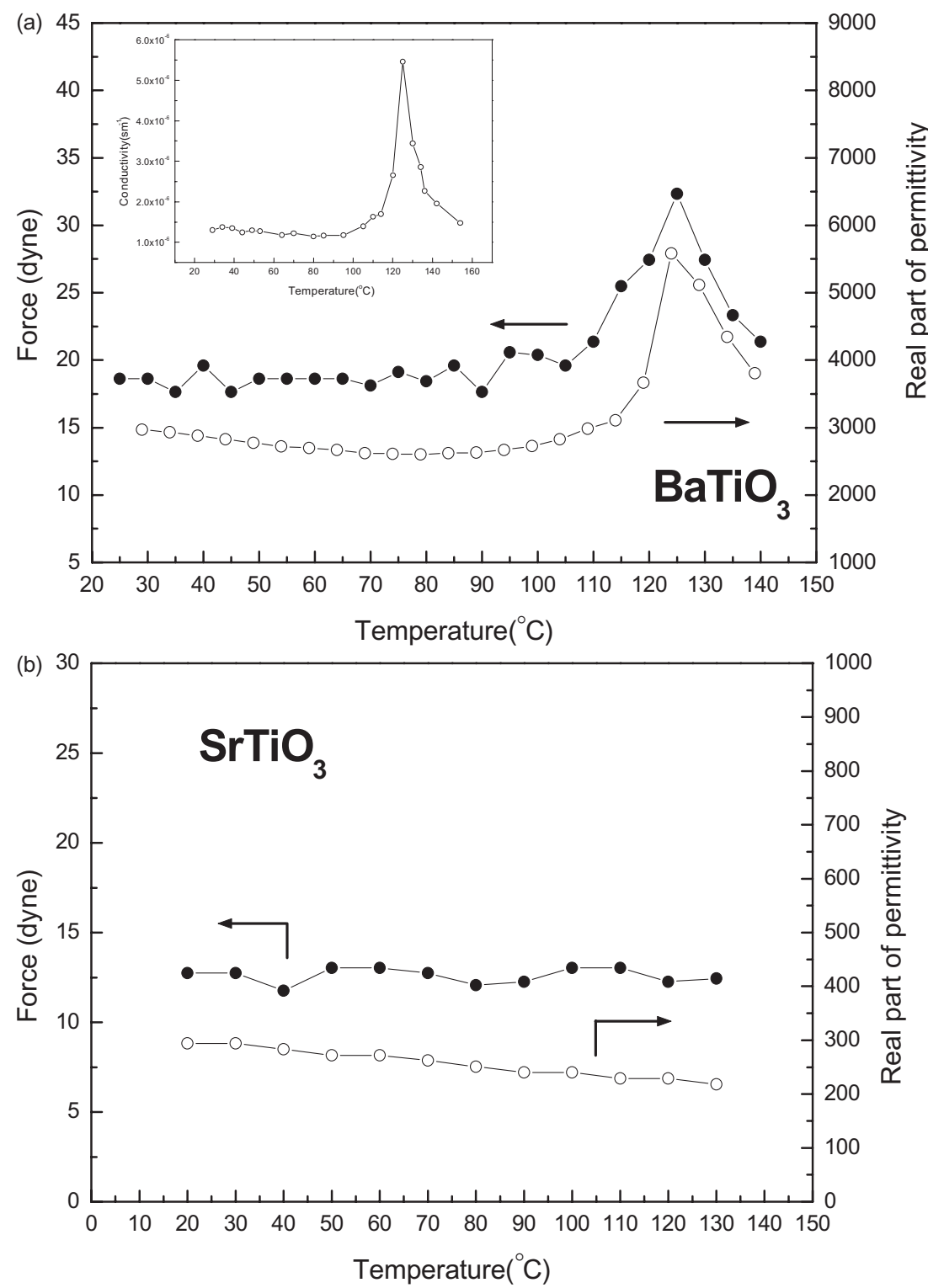

Figure 1. Variations in the induced force and the real part of the permittivity versus temperature: $(a) \mathrm{BaTiO}_{3}$, the inset gives the temperature dependence of its conductivity; (b) $\mathrm{SrTiO}_{3}$.

accuracy within $\pm 1^{\circ} \mathrm{C}$. The average temperature difference between the thermocouple spot and the samples was less than $1{ }^{\circ} \mathrm{C}$. All the experimental samples were heated at $100^{\circ} \mathrm{C}$ for $24 \mathrm{~h}$ to remove water before measuring. At each experimental point we collected the data 3-4 times, and then we got the average. The maximum of the average deviation was $1 \mathrm{mg}$ for the measured forces. The details of the experimental set-up used for measurement of the force can be found in our previous papers $[10,11]$. The whole apparatus was sealed in a Plexiglas box for protection from moisture and dust.

\section{Results and discussion}

The temperature dependence of the dielectric constant and the corresponding interaction force of the spheres for the $\mathrm{BaTiO}_{3}$ sample in silicone oil $\left(\varepsilon=2.54, \sigma=10^{-13} \mathrm{~S} \mathrm{~m}^{-1}\right)$ are shown in figure $1(a)$. We note that the obvious peaks of the dielectric constant and conductivity (shown in the inset of figure $1(a)$ ) could be identified at the Curie temperature, $T_{\mathrm{c}} \sim 125^{\circ} \mathrm{C}$, for $\mathrm{BaTiO}_{3}$ and, correspondingly, the interspherical force reached its maximum; here the frequency and field strength were, respectively, fixed at $200 \mathrm{~Hz}$ and $50.8 \mathrm{~V} \mathrm{~mm}^{-1}$ and the gap spacing, $\delta$, between two spheres was $0.01 \mathrm{~mm}$. We attributed this increase in the interaction force solely to the changes in the dielectric constant since the contribution of its conductivity could be ignored due to its relatively smaller value. The increase in the conductivity of the silicone oil, $\sigma_{\mathrm{oil}}$, when the temperature varied from room temperature to $125^{\circ} \mathrm{C}$, was also neglected (its value is around $10^{-12} \mathrm{~S} \mathrm{~m}^{-1}$ at room temperature and will increase slightly with temperature [12]). According to

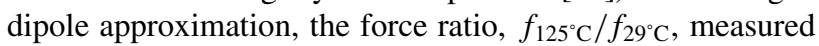
at two temperatures is proportional to that of the mismatched factor ratio, $\beta_{125^{\circ} \mathrm{C}}^{2} / \beta_{29^{\circ} \mathrm{C}}^{2}$, which is calculated to be about 1 . However, our experimental result is $f_{125^{\circ} \mathrm{C}} / f_{29^{\circ} \mathrm{C}}=1.74$, which is in disagreement with the result derived using the 


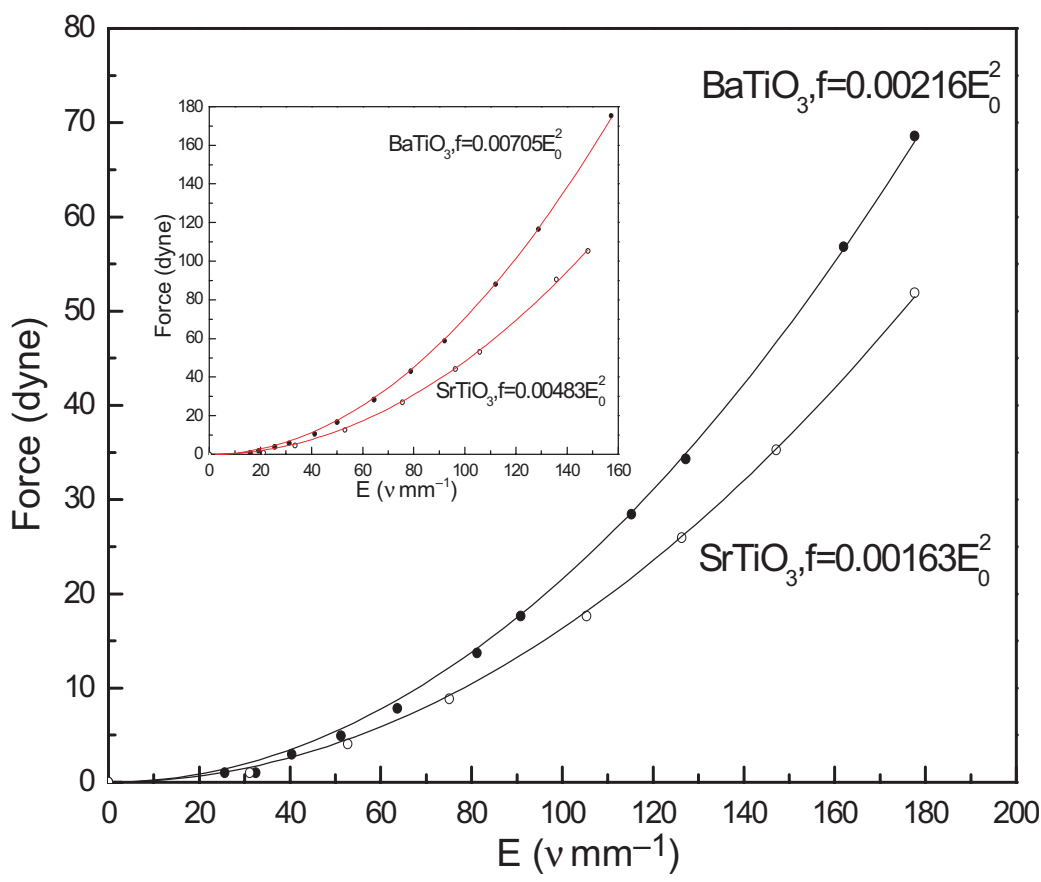

Figure 2. Comparison of the induced interspherical forces of $\mathrm{BaTiO}_{3}$ and $\mathrm{SrTiO}_{3}$ spheres filled with silicone oil under a $50 \mathrm{~Hz}$ ac electric field: here $\delta=0.05 \mathrm{~mm}$, while in the inset $\delta=0.01 \mathrm{~mm}$. The solid lines are the fitted curves.

simple dipole model. Figure $1(b)$ shows that, when $\mathrm{BaTiO}_{3}$ spheres were replaced with $\mathrm{SrTiO}_{3}$ spheres but all other experimental conditions remained unchanged, there was no abrupt change in either the dielectric constant or the interaction force among the spheres, which indicates that the abnormal changes in the interaction force were indeed the result of changes in the dielectric properties of the materials as the temperature increased.

The field dependences of the respective interspherical forces of $\mathrm{BaTiO}_{3}$ and $\mathrm{SrTiO}_{3}\left(\varepsilon_{\mathrm{p}}=294, \sigma_{\mathrm{p}}=2 \times 10^{-8} \mathrm{~S} \mathrm{~m}^{-1}\right)$ spheres in silicone oil at room temperature are shown in figure 2 , where the relation $f \sim E_{0}^{2}$ is satisfied. When the gap spacing of two spheres decreases from 0.05 to $0.01 \mathrm{~mm}$, the interaction force correspondingly increases, which can be seen in the inset of figure 2. From figure 2, we calculated from the experimental results that the force ratios, $f_{\mathrm{BaTiO}_{3}} / f_{\mathrm{SrTiO}_{3}}$, are 1.33 and 1.46 for the cases of the two gaps, which are both larger than the calculated value of 1.06 according to the dipole approximation. Further, we found that the longer the distance between the two spheres, was the closer the agreement was between theory and experiment.

In the past we observed that the force, $f$, hardly increases with the dielectric constant, $\varepsilon_{\mathrm{p}}$, increasing due to the dielectric mismatch of high dielectric spheres/low dielectric medium tends to be 1 . The above experimental results prove that the interaction of higher dielectric spheres is still stronger under an electric field of lower frequency, showing that the dielectric mismatch is not sufficiently sensitive to quantitatively describe the interaction force of high dielectric materials. The local field effect, $E_{\text {local }}$, is considered to explain our results. We compare the interspherical forces of two systems whose dielectric mismatch factors are 0.98 and 0.97 , respectively.
With the centre-to-centre line of two spheres parallel to the applied field vector, $E_{0}$, and the sphere radius $R \gg \delta$, the earlier literature gave the local field modification $E_{\text {local }}=$ $E_{0} /(1-0.60 \beta)^{0.4}[7,13]$. The real $E_{\text {local }}\left[E_{0} /(1-\beta)^{0.4}\right]$ is actually stronger than the theoretical $E_{\text {local }}[11]$. According to the point dipolar model, the force ratio $f_{0.98} / f_{0.97}$ is equal to 1.02 ; however, the ratios are 1.05 and 1.38 , respectively, based on the above theoretical modification and experimental modification. It is obvious that the local effect has a great influence on the contributions of $\varepsilon_{\mathrm{p}}$ to the interspherical force.

We also checked the influence of the surrounding medium on the interaction forces between two identical $\mathrm{BaTiO}_{3}$ spheres at room temperature, and the results are shown in figure 3 , where two kinds of media, silicone oil and dry air, were chosen. The electric field frequency is fixed to be $150 \mathrm{~Hz}$. Since $\varepsilon_{\mathrm{BaTiO}_{3}} \gg \varepsilon_{\text {air }}, \varepsilon_{\mathrm{BaTiO}_{3}} \gg \varepsilon_{\text {oil }}$ and $\sigma_{\mathrm{BaTiO}_{3}} \gg \sigma_{\text {air }}, \sigma_{\mathrm{BaTiO}_{3}} \gg$ $\sigma_{\text {oil }}$, we are able to assume that the two systems have approximately same dielectric mismatch [8]. Thus, we can deduce that $f_{\text {oil }} / f_{\text {air }}$ is equal to $\varepsilon_{\text {oil }} / \varepsilon_{\text {air }}(2.54 / 1)$, while the local field of a sphere does not influence its neighbour. We note that the interaction is indeed much stronger when the spheres are surrounded by silicone oil. As shown in figure 3 and the inset, respectively, the measured force in silicone oil is 2.45 times stronger than the measured force in gas for the gap of $0.05 \mathrm{~mm}$ and is 2.95 times the latter for a $0.01 \mathrm{~mm}$ gap. The results for the $0.05 \mathrm{~mm}$ spaced spheres are in accordance with the deduction due to the weaker local field effect.

The frequency dependences of the interspherical force, $f$, and the square mismatch factor, $\beta^{2}$, of $\mathrm{BaTiO}_{3}$ spheres in silicone oil at room temperature are shown in figure 4 , where the corresponding dielectric properties are plotted in 


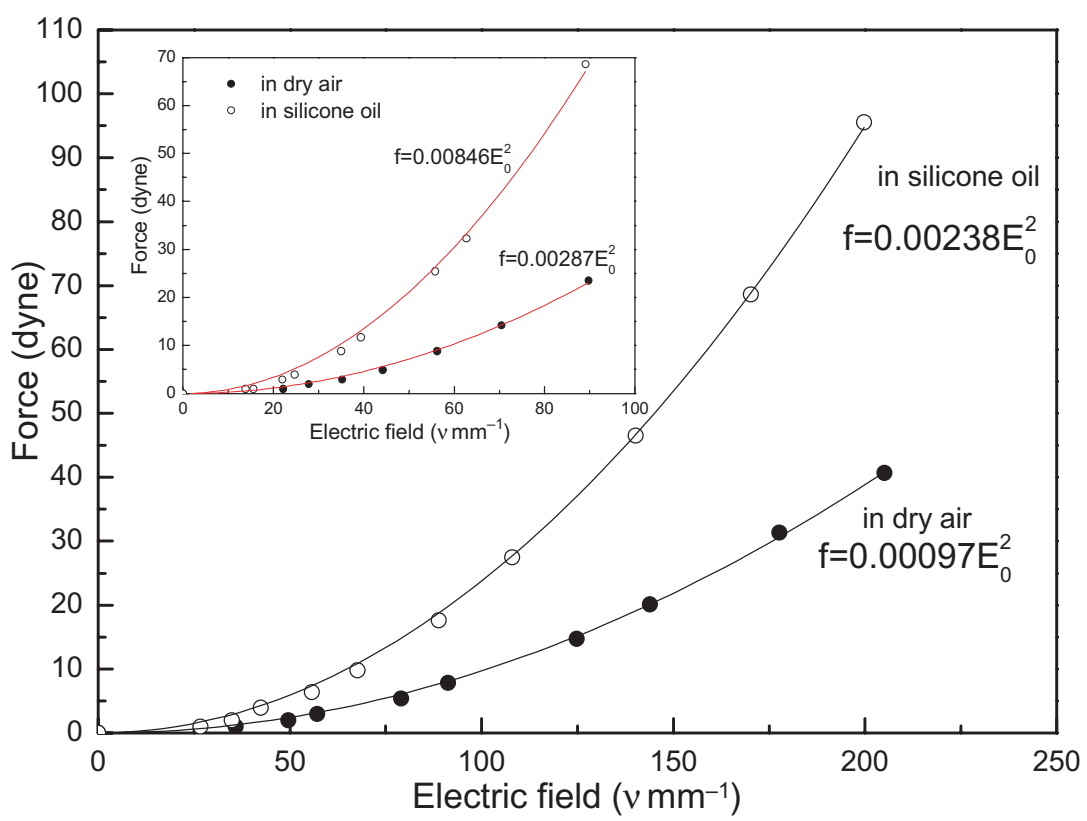

Figure 3. Induced forces of two $\mathrm{BaTiO}_{3}$ spheres in two kinds of media, silicone oil and dry air. Here $\delta=0.05 \mathrm{~mm}$, while in the inset $\delta=0.01 \mathrm{~mm}$. The solid lines are the fitted curves.

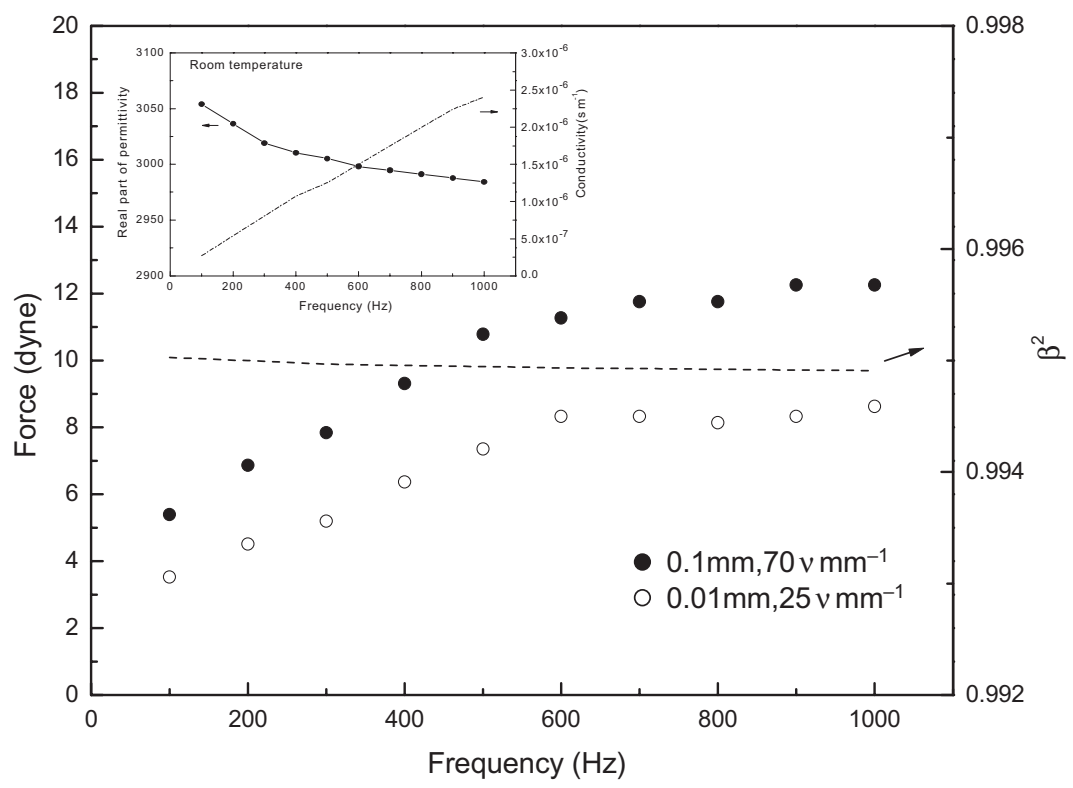

Figure 4. Variations of the induced forces of two $\mathrm{BaTiO}_{3}$ spheres with respect to the field frequency at $\delta=0.1 \mathrm{~mm}, E_{0}=70 \mathrm{~V} \mathrm{~mm}{ }^{-1}$ and $\delta=0.01 \mathrm{~mm}, E_{0}=25 \mathrm{~V} \mathrm{~mm}^{-1}(\mathrm{O})$. The dashed line represents the calculated value of $\beta^{2}$ versus frequency. The inset shows the frequency dependence of the dielectric properties of $\mathrm{BaTiO}_{3}$.

the inset. From the figure, we note that the value of $\beta^{2}$ is almost unchanged in the frequency range investigated while the interaction forces measured with different gaps increase. This is a puzzle if the dipole model is employed to explain these experimental observations since the interaction force, $f$, is usually proportional to $\beta^{2}$. We think that the disagreement between the experiment and theory based on the dipole assumption must result from some other factors. Therefore, when the spheres are closely spaced, the simple dipolar model is no longer valid [5].

\section{Conclusions}

The interaction force between dielectric spheres is strongly associated with the spheres' dielectric properties. The tendency is that the higher the dielectric constant is, the stronger the interaction force is. The quadratic relationships between the interaction force and field strength have been identified for both $\mathrm{BaTiO}_{3}$ and $\mathrm{SrTiO}_{3}$ spheres. We found from experiments that the surrounding medium is important in influencing the interaction of the spheres. Our experimental 
results indicate that the local effect should be taken into account in discussing the contributions of $\varepsilon_{\mathrm{p}}$ and $\varepsilon_{\mathrm{m}}$ to the interspherical force. In addition, the interaction force is dependent on the frequency of the field.

\section{Acknowledgments}

This research was supported by the National Science Natural Foundation of China, No 10029401 and RGC HKUST 6065/02P. We would like to thank Professors Yaqin Zhao and Meiying Hou for their assistance in preparing this paper.

\section{References}

[1] Winslow W M 1949 J. Appl. Phys. 201137

[2] Klass D L and Martinek T W 1967 J. Appl. Phys. 3867
[3] Block H and Kelly J P 1988 J. Phys. D: Appl. Phys. 21 1661

[4] Coulson C A 1961 Electricity (New York: Wiley) p 42

[5] Chen Y, Sprecher A F and Conrad H 1991 J. Appl. Phys. 70 6796

[6] Davis L C 1992 Appl. Phys. Lett. 60319

[7] Tao R, Jiang Q and Sim H K 1995 Phys. Rev. E 52 2727

[8] Lu K Q, Wen W J, Li C X and Xie S C 1995 Phys. Rev. E 52 6329

[9] Lan Y C, Men S Q, Zhao X P and Lu K Q 1998 Appl. Phys. Lett. 72653

[10] Wang Z Y, Peng Z, Lu K Q and Wen W J 2003 Appl. Phys. Lett. 821796

[11] Wang Z Y, Shen R, Niu X J, Lu K Q and Wen W J 2003 J. Appl. Phys. 947832

[12] Wu C and Conrad H 1997 J. Phys. D: Appl. Phys. 30 2634

[13] Davis L C 1992 J. Appl. Phys. 721334 